\title{
Comparação das atividades básicas de vida diária entre idosos hospitalizados e hígidos da região sul do Brasil
}

\author{
Functional capacity of hospitalized elderly people in a hospital \\ of the south region of Brazil
}

Mônica Wietzke, ${ }^{1}$ Marciele Silveira Hopp, ${ }^{1}$ Litiele Evelin Wagner, ${ }^{1}$ João Guilherme dos Santos Fagundes, ${ }^{1}$ Dannuey Machado Cardoso, ${ }^{1}$ Dulciane Nunes Paiva ${ }^{1}$

Universidade de Santa Cruz do Sul (Unisc), Santa Cruz do Sul, RS, Brasil.

Recebido em: 05/04/2017 / Aceito em: 07/08/2017 / Publicado em: 01/10/2017

dulciane@unisc.br

\section{RESUMO}

Objetivo: comparar as atividades básicas de vida diária (ABVDs), entre idosos hospitalizados e hígidos. Método: estudo transversal que avaliou 51 idosos de ambos os sexos hospitalizados, sob condição não cirúrgica (Grupo Hospitalizado - $\mathrm{GH}, \mathrm{n}=16$ ) e idosos hígidos (Grupo Controle - GC, $\mathrm{n}=35$ ), no período de março a junho de 2013. As ABVDs foram avaliadas através do questionário Basic Activities of Daily Living (BADL), que mensura o nível de independência em seis atividades básicas de vida diária. Comparação entre grupos quanto às características antropométricas avaliadas através do teste $t$ Student, independente ou o teste $U$ de Mann-Whitney. Proporções das variáveis categóricas comparadas pelo teste de Qui-quadrado $(p<0,05)$. Resultados: amostra $(n=51)$, com idade

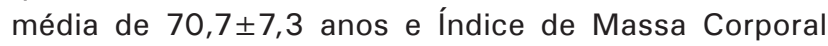
(IMC) de $26,2 \pm 3,6 \mathrm{Kg} / \mathrm{m}^{2}$. A mediana do tempo total de internação e do tempo de internação até a data da avaliação dos pacientes foi de 13 (6-64) e 7 (2-31) dias, respectivamente. Houve diferença significativa no escore na Escala BADL, obtido no $\mathrm{GH}(4,6 \pm 1,8)$ e no $\operatorname{GC}(5,8 \pm 0,3)(p=0,008)$. Considerações finais: os idosos hospitalizados apresentaram menor escore das ABVDs, tendo sido evidenciada diferença significativa dos domínios avaliados em relação aos idosos hígidos.

Palavras-chave: Envelhecimento; Institucionalização; Hospitalização; Morbidade.

\section{ABSTRACT}

Objective: to compare the basic activities of daily living (BADL) among hospitalized and healthy elderly. Methods: a cross-sectional study that evaluated the elderly of both sexes in the period from march to june 2013, hospitalized under non-surgical conditions (Hospitalized Group - HG, $n=16$ ) and healthy elderly (Control Group - CG, $n=35$ ). The BADL was assessed using the Basic Activities Scale of Daily Living (BADL) questionnaire, which measures the level of independence in six activities of daily living. Results: sample $(n=$ 51) with mean age of $70.7 \pm 7.3$ years and Body Mass Index (BMI) of $26.2 \pm 3.6 \mathrm{~kg} / \mathrm{m}^{2}$. The median time of hospitalization and length of hospital stay up to the date of the patients' evaluation was 13 (6-64) and 7 (2-31) days, respectively. There was a significant difference

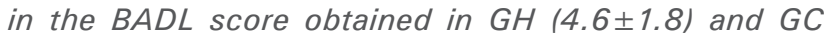
(5.8 \pm 0.3$)(p=0.008)$. Closing remarks: hospitalized elderly had a lower BADL score and a significant difference was observed in the domains evaluated in relation to healthy elderly subjects.

Keywords: Aging; Institutionalization; Hospitalization; Morbidity. 


\section{INTRODUÇÃO}

O envelhecimento populacional ocasiona alterações fisiológicas que tornam os indivíduos mais suscetíveis às doenças crônicas, o que pode impactar na capacidade de realização das Atividades Básicas de Vida Diária (ABVDs) e acelerar sua vulnerabilidade. ${ }^{1-3}$

A existência de doença de base no idoso associada à internação hospitalar e ao próprio envelhecimento aumenta a morbidade. ${ }^{4}$ A hospitalização é um evento crítico na vida dos idosos e acarreta implicações, como mudanças na qualidade de vida, declínio funcional (que atinge de $25 \%$ a $35 \%$ dos idosos hospitalizados), ${ }^{5}$ institucionalização e até a morte, tendo a deficiência funcional se tornado um forte indicador de morbidade e mortalidade em idosos. ${ }^{6,7}$ Nesse sentido, a capacidade funcional (CF) é definida como a realização de atividades relacionadas à sobrevivência de forma autônoma e independente. ${ }^{8}$

A CF é a base de uma avaliação geriátrica eficiente e permite classificar os idosos quanto aos níveis de realização de suas atividades, ${ }^{8}$ utilizando escalas de dificuldade e dependência que buscam avaliar o desempenho do indivíduo, através da execução de tarefas desenvolvidas em um ambiente habitual. Tais escalas assumem três formas padrão: (i) o grau de dificuldade (nenhuma até muita dificuldade), (ii) o grau de assistência ou de dependência (independente/sem assistência a total dependência/assistência) e (iii) se a atividade é realizada (realiza ou incapaz), abrangendo as ABVDs, as Atividades Instrumentais da Vida Diária (AIVDs) e as Atividades Avançadas de Vida Diária (AAVDs). Ressalta-se que a dificuldade de realização das ABVDs, das AIVDs e da mobilidade são os indicadores mais utilizados em estudos epidemiológicos, para avaliar a incapacidade. ${ }^{9}$

Faz-se importante a avaliação do status funcional dos idosos durante o período de hospitalização, pois tal fato provoca danos em sua autonomia e aumenta a probabilidade de incapacidade funcional, o que pode interferir em sua morbidade bem como em suas ABVDs. ${ }^{10,11}$ Nesse sentido, o presente estudo objetivou quantificar e comparar as ABVDs entre idosos hospitalizados e hígidos.

\section{MÉTODO}

Trata-se de estudo transversal desenvolvido no período de março a junho de 2013 que avaliou 51 idosos de ambos os sexos alocados no Grupo Controle (GC) e no Grupo Hospitalizado (GH). Os idosos hospitalizados foram triados a partir de revisão dos seus prontuários nas enfermarias do Hospital Santa Cruz do Sul (HSC) Santa Cruz do Sul - RS. Os idosos alocados no GC foram triados no Grupo de Convivência de ldosos do Complexo Poliesportivo da região, tendo o estudo sido aprovado pelo Comitê de Ética e Pesquisa com Seres Humanos da Universidade de Santa Cruz do Sul (CAAE - 08015213.6.00005343; Parecer $n^{\circ}$ 218.439).

Foram incluídos idosos hospitalizados com perfil não cirúrgico, admitidos por eventos agudos ou crônicos agudizados e com tempo de internação maior que 48 horas e, excluídos aqueles com IMC $>40 \mathrm{Kg} / \mathrm{m}^{2}$, diagnóstico de doença pulmonar aguda ou crônica, instabilidade hemodinâmica, depressão do sensório ou outras neuropatias que comprometessem a cognição e, ainda, aqueles submetidos a procedimentos cirúrgicos ou com instabilidade clínica. Para compor o GC, foram incluídos os idosos insuficientemente ativos de acordo com a classificação do Questionário Internacional de Atividade Física (IPAQ), excluídos os praticantes de exercício físico regular ou que tivessem apresentado sintomas respiratórios no período de 30 dias antecedentes à avaliação (Figura 1).

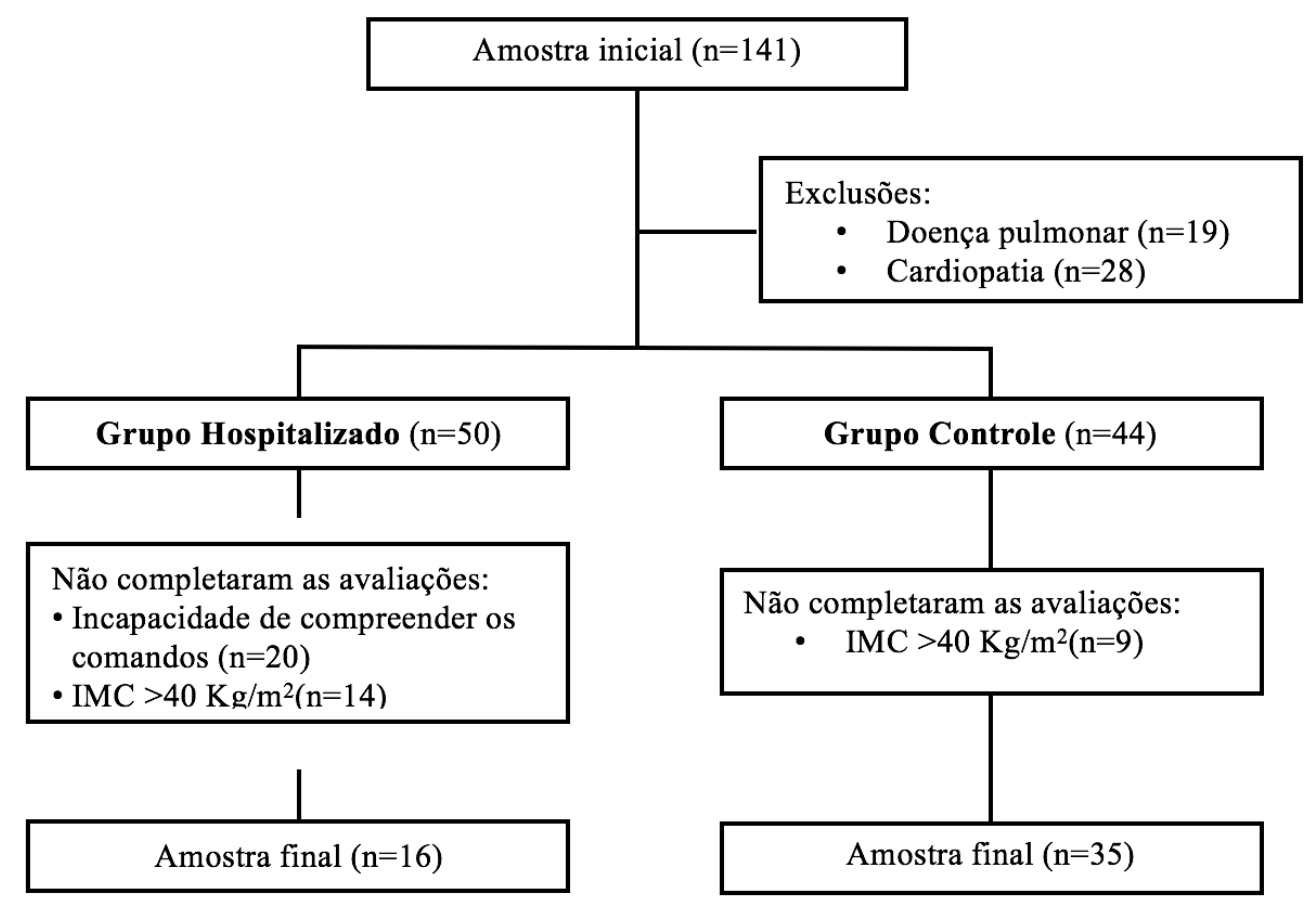

Figura 1 - Fluxograma do estudo. 
Foram avaliadas a pressão arterial sistólica (PAS) e diastólica (PAD) (PREMIUM ${ }^{\oplus}$, modelo ESFHS50, Brasil), a frequência respiratória (FR), a frequência cardíaca (FC), temperatura axilar (Tax) e a saturação periférica de oxigênio $\left(\mathrm{SpO}_{2}\right)$ (Nonin ${ }^{\oplus}$, modelo Onix 9500, Winsconsin, EUA) e imediatamente após foi avaliada a CF. Para estimativa do peso, altura e posterior cálculo de Índice de Massa Corporal (IMC) foi utilizada a fórmula preconizada por Chumlea, Roche e Steinbaugh ${ }^{12}$ Para identificar o nível de atividade física dos pacientes idosos avaliados foi utilizado o IPAQ (Versão Curta), sendo a interpretação desse instrumento baseada nas recomendações do Centro de Estudos Laboratório de Aptidão Física de São Caetano (CELAFISCS). ${ }^{13}$

\section{Capacidade funcional (CF)}

Para avaliar a CF foi utilizada a Escala Basic Activities of Daily Living (BADL), ${ }^{14}$ que consiste em um questionário destinado a mensurar a independência em seis AVDs (banhar-se, vestir-se, ir ao banheiro, transferências, continência e alimentação). Nesse instrumento autorreferido, as atividades foram avaliadas, quanto ao grau de dependência ou independência em toda sua execução, desde seu princípio até sua conclusão. Caso o indivíduo consiga realizar as tarefas soma-se um ponto na escala e caso contrário, pontua-se com zero. Ao final, somam-se todas as atividades para a obtenção da seguinte classificação: 'independente' (6 pontos), 'parcialmente dependente' (4 pontos) e 'totalmente dependente' (2 pontos ou menos).

\section{Análise estatística}

Foi utilizado o programa Statistical Package for the Social Sciences (SPSS, versão 20.0) para verificar a normalidade da distribuição dos dados através do teste de Shapiro-Wilk, sendo os resultados expressos em média e desvio padrão ou mediana com intervalo interquartil. Para comparação entre os grupos, quanto às características antropométricas foi utilizado o teste t Student independente ou o teste $U$ de Mann-Whitney. Proporções das variáveis categóricas foram comparadas pelo teste de Qui-quadrado $(p<0,05)$.

\section{RESULTADOS}

A amostra foi composta por 51 indivíduos $(\mathrm{GH}$ : $\mathrm{n}=16 ; \mathrm{GC}: \mathrm{n}=35$ ) com média de idade de $70,7 \pm 7,3$ anos. Na tabela 1 podem ser observadas as características dos grupos analisados. A mediana do tempo total de internação e do tempo de internação até a data da avaliação dos pacientes foi de 13 (6-64) e 7 (2-31) dias, respectivamente.

Houve diferença significativa do escore da escala BADL entre $\mathrm{GH}(4,6 \pm 1,8)$ e $\mathrm{GC}(5,8 \pm 0,3) \quad(p=0,008)$ (Figura 2).

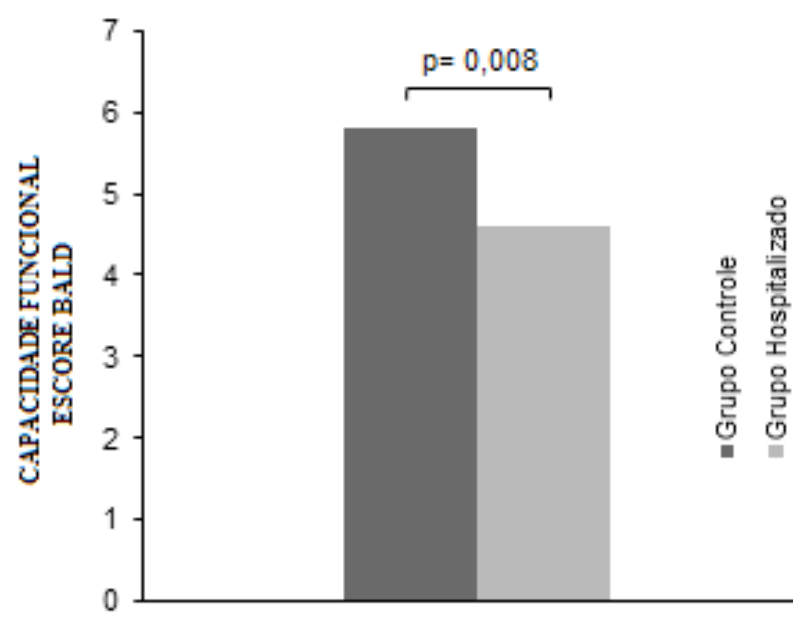

Figura 2 - Comparação da Capacidade Funcional entre o Grupo Hospitalizado e o Grupo Controle.

Tabela 1 -Caracterização da amostra avaliada.

\begin{tabular}{|c|c|c|c|}
\hline & $\mathrm{GH}(n=16)$ & $\mathrm{GC}(\mathrm{n}=35)$ & p-valor \\
\hline Sexo masculino, n (\%) & $10(62,5)$ & $22(62,9)$ & 0,609 \\
\hline Idade (anos)* & $73(68,6-81,2)$ & $69(64-73)$ & 0,095 \\
\hline Peso $(\mathrm{Kg}) * *$ & $63,9 \pm 13,8$ & $71,1 \pm 11,5$ & 0,062 \\
\hline Altura $(\mathrm{cm}) * *$ & $157,5 \pm 8,9$ & $161,7 \pm 8,3$ & 0,117 \\
\hline IMC $\left(\mathrm{Kg} / \mathrm{m}^{2}\right)^{* *}$ & $25,3 \pm 4,2$ & $26,6 \pm 3,2$ & 0,214 \\
\hline $\mathrm{SpO} 2(\%) *$ & $95,5(93-97)$ & $97(95-98)$ & 0,041 \\
\hline \multicolumn{4}{|l|}{ Sinas Vitais } \\
\hline PAS $(\mathrm{mmHg})^{*}$ & $135(122,5-140)$ & $130(120-130)$ & 0,174 \\
\hline $\operatorname{PAD}(\mathrm{mmHg})^{*}$ & $80(70-90)$ & $80(70-87,5)$ & 0,899 \\
\hline $\mathrm{FR}\left(\right.$ irpm) ${ }^{* *}$ & $19,3 \pm 3,8$ & $18,6 \pm 3,4$ & 0,535 \\
\hline $\mathrm{FC}(\mathrm{bpm})^{*}$ & $73(68,2-81,2)$ & $77(72-81)$ & 0,158 \\
\hline $\operatorname{Tax}(\mathrm{oC}) *$ & $36(36-37)$ & $35(35-37)$ & $<0,001$ \\
\hline \multicolumn{4}{|l|}{ Tabagistas } \\
\hline Ativos & $2(12,5)$ & $3(8,6)$ & 0,844 \\
\hline \multicolumn{4}{|l|}{ Uso de Medicamentos } \\
\hline Diuréticos & $3(18,8)$ & $4(11,4)$ & 0,664 \\
\hline Sedativos & $2(5,7)$ & $1(6,2)$ & 0,940 \\
\hline
\end{tabular}

IMC: Índice de Massa Corporal; PAS: Pressão Arterial Sistêmica; PAD: Pressão Arterial Diastólica; FR: Frequência Respiratória; FC: Frequência Cardíaca; SpO2: Saturação Periférica de Oxigênio; Tax: Temperatura Axilar; " valores em mediana e intervalo interquartil (Teste U de Mann-Whitney). * *Média e desvio padrão (teste t Student). Valores significativos com $p<0,05$. 


\section{DISCUS5ÃO}

O processo de envelhecimento populacional ocorre de forma muito mais acelerada nos países emergentes e subdesenvolvidos e as características inerentes a esse processo tornam os idosos mais susceptíveis às doenças crônicas e complicações que podem conduzir à internação hospitalar e, nesse contexto, à perda da capacidade funcional pode ser consequente aos eventos adversos que podem ocorrer, durante e após o processo de internação hospitalar. ${ }^{15}$ Assim, o presente estudo evidenciou que idosos hospitalizados apresentaram redução dos níveis de realização de suas ABVDs em relação aos idosos hígidos que apresentavam as mesmas características antropométricas.

A hospitalização de idosos usualmente ocorre devido ao desenvolvimento de doenças que requerem cuidados especiais e que resultam em maiores chances de surgimento de incapacidade funcional, em que as mesmas impactam diretamente sobre a qualidade de vida do idoso, podendo ocasionar consequências irreversíveis por impor graus variados de imobilidade..$^{6,8}$

Em estudo que avaliou a CF de idosos internados em um serviço de emergência constatou-se que dos 200 idosos avaliados, $65 \%$ eram independentes, $11 \%$ parcialmente dependentes e $25 \%$ dependentes, sendo que idosos mais velhos, do sexo feminino, com doenças cerebrovasculares e demência apresentaram maior dependência funcional, ${ }^{11}$ segundo Lopes et al. ${ }^{16}$ Além disso, a restrição ao leito desencadeia modificações na musculatura e na massa óssea, ocasionando restrições no desenvolvimento das AVDs dos idosos. ${ }^{6}$ As manifestações iniciais afetam diretamente o sistema musculoesquelético, alterando a força muscular, a resistência à fadiga e o vigor físico.

Vários fatores podem contribuir para a perda de capacidade de realização das ABVDs em idosos hospitalizados como o desenvolvimento de processos infecciosos, hipertensão arterial sistêmica e o tempo de internação hospitalar. ${ }^{16} \mathrm{Em}$ nosso estudo, a mediana do tempo total de internação e do tempo de internação, até a data da avaliação dos pacientes foi de 13 (6-64) e 7 (2-31) dias, que é considerado, segundo a média nacional (que é de 6,6 dias), uma permanência hospitalar prolongada. ${ }^{16}$ A permanência hospitalar prolongada (PHP) é um indicador indireto da qualidade do cuidado prestado aos pacientes nos serviços terciários embora essa relação não esteja bem estabelecida. ${ }^{17,18}$

Kortebein et al. ${ }^{19}$ afirmam que a restrição ao leito associado à inatividade física induz os pacientes a um declínio da CF devido à perda de força dos membros inferiores e redução da capacidade aeróbica. Ao utilizar a medida de independência funcional (MIF) em 116 idosos longevos internados em unidades hospitalares em Curitiba - PR, Lourenço et al. ${ }^{19}$ encontraram valores que representam independência funcional, entretanto, os idosos avaliados apresentaram dificuldade na realização de determinadas atividades como controle de esfíncteres, transferências entre cama, cadeira, cadeira de rodas, vaso sanitário, chuveiro/banheira e locomoção em escadas. ${ }^{20}$

Deve ser ressaltado que o presente estudo apresentou algumas limitações como o fato de não ter sido possível avaliar os idosos na condição pré-hospitalização, além do fato da amostra avaliada ter sido composta por indivíduos com diferentes condições clínicas. A escassez de estudos que envolvem o idoso hospitalizado sob condição não cirúrgica não possibilitou ampliar a discussão com os dados obtidos.

Ressalta-se que a identificação das características funcionais dos idosos é de extrema importância para uma abordagem especializada e que vise à identificação de problemas clínicos, permitindo uma melhor qualidade no atendimento e emprego mais adequado dos recursos disponíveis dentro da realidade existente. Torna-se importante reconhecer as incapacidades funcionais que provocam danos na autonomia dos pacientes idosos, pois as mesmas podem interferir em suas AVDs e qualidade de vida.

\section{CONSIDERAÇÕES FINAIS}

Em conclusão, nossos dados apontam a existência de dependência funcional em pacientes sob a condição de hospitalização, tendo os idosos hospitalizados apresentado redução na capacidade de realização de suas atividades básicas de vida diária. Tal resultado remete à necessidade do reconhecimento precoce das características desses indivíduos para que se possa estabelecer uma abordagem especializada adequada, visando à identificação de problemas que venham a acometer o idoso durante o período de hospitalização.

\section{REFERÊNCIAS}

1. Tenório DM, Camacho AC. Identificação dos agravos de saúde que levam os Idosos ao serviço de emergência. Rev Enferm UFPE On Line 2015;9(supl.1):457-465. doi: 10.5205/ reuol.5221-43270-1-RV.0901supl201526

2. Nogueira SL, Ribeiro RC, Rosado LE, Franceschini SC, Ribeiro $A Q$, Pereira ET. Fatores determinantes da capacidade funcional em idosos longevos. Rev Bras Fisioter 2010;14(4):322-329. doi: 10.1590/S1413-35552010005000019

3. Storti LB, Fabrício-Whebe SC, Kusumota L, Rodrigues RA, Marques S. Fragilidade de idosos internados na clínica médica da unidade de emergência de um hospital geral terciário. Texto contexto - Enferm 2013;22(2):452-459. doi: 10.1590/ S0104-07072013000200022

4. Lee LC, Tsai AC. Mini-Nutritional-Assessment (MNA) without Body Mass Index (BMI) predicts functional disability in elderly Taiwanese. Arch Gerontol Geriatr 2012;54(3):e405-410. doi: 10.1016/j.archger.2011.12.006

5. Pereira CAC, Sato Taeko, Rodrigues CS. Novos valores de referência para espirometria forçada em brasileiros adultos de raça branca. J Bras Pneumol 2007;33(4):397-406. doi: 10.1590/S1806-37132007000400008

6. Peron EP, Gray SL, Hanlon JT. Medication use and functional status decline in older adults: a narrative review. Am J Geriatr Pharmacother 2011;9(6):378-391. doi: 10.1016/j.amjopharm.2011.10.002

7. Buurman BM, van Munster BC, Korevaar JC, de Haan RJ, de Rooij SE. Variability in measuring (instrumental) activities of daily living functioning and functional decline in hospitalized older medical patients: a systematic review. J Clin Epidemiol 2011;64(4):619-627. doi: 10.1016/j.jclinepi.2010.07.005

8. Freitas RS, Fernandes $\mathrm{MH}$, Coqueiro RDS, et al. Capacidade 
funcional e fatores associados em idosos: estudo populacional. Acta Paul Enferm 2012;25(6). doi:10.1590/ S0103-21002012000600017

9. Costa FAM, Silva AM, Amorim FTR, et al. Contribuição das doenças crônicas na prevalência da incapacidade para as atividades básicas (ABVD) e instrumentais (AIVD) de vida diária entre idoso brasileiros. Belo Horizonte. Dissertação [Mestrado em Saúde Coletiva]. Fundação Oswaldo Cruz 2016.

10. Roberts HC, Denison HJ, Martin HJ, Patel HP, Syddall $\mathrm{H}$, Cooper $\mathrm{C}$, et al. A review of the measurement of grip strength in clinical and epidemiological studies: towards a standardised approach. Age Ageing 2011;40(4):423-429. doi: 10.1093/ageing/afr051

11. Freitas $R \mathrm{~S}$, Fernandes $M H$, Coqueiro $R D S$, Reis Júnior W M, Rocha S V, Brito T A. Capacidade funcional e fatores associados em idosos: estudo populacional. Acta Paul Enferm 2012;25(6):933-939. Disponível em: http://www. scielo.br/pdf/ape/v25n6/v25n6a17.pdf

12. Chumlea WC, Roche AF, Steinbaugh ML. Estimating stature from kneeheight for persons 60 to 90 years of age. J Am Geriatr Soc 1985;33(2):116-120. doi: 10.1111/j.15325415.1985.tb02276.x.

13. Dawalibi NW, Goulart RMM, Prearo LC. Fatores relacionados à qualidade de vida de idosos em programas para a terceira idade. Cien Saúde Colet 2014;19(8):3505-3512. doi: 10.1590/1413-81232014198.21242013

14. Andrade NB, Novelli MMPC. Perfil cognitivo e funcional de idosos frequentadores dos centros de convivência para idosos da cidade de Santos. Cad. Ter. Ocup 2015;23(1):143 152. doi: dx.doi.org/10.4322\%2F0104-4931.ctoAO396

15. Fátima de Lima Paula, Geraldo Marcelo da Cunha, lúri da
Costa Leite, Rejane Sobrino Pinheiro, Joaquim Gonçalves Valente. Readmissão de idosos por fratura proximal do fêmur: uma abordagem multinível. Rev Saúde Pública 2016;50:16. Disponível em: http://www.redalyc.org/ pdf/672/67247719013.pdf

16. Lopes MCBT, Lagel JSS, Vancini-Campanharo CR, Okumo MFP, Batista REA. Fatores associados ao comprometimento funcional de idosos internados no serviço de emergência. Einstein 2015;13(2):209-214. doi: 10.1590/S167945082015А03327.

17. Geísa Pereira Rufino, Mariana Galvão Gurgel, Thaís de Carvalho Pontes, Eutília Freire. Avaliação de fatores determinantes do tempo de internação em clínica médica. Rev Bras Clin Med São Paulo 2012;10(4):291-297. Disponível em: http://files.bvs.br/ upload/S/1679-1010/2012/v10n4/a3043.pdf

18. Coventry LL, Pickles S, Sin M, Towell A, Giles M, Murray K, Twigg D. Impact of the orthopaedic nurse practitioner role on acute hospital length of Stay and cost-savings for patients with hip fracture: A retrospective cohort study. J Adv Nurs 2017;73(11):2652-63. doi: 10.1111/jan.13330.

19. Kortebein $P$, Symons $T$ B, Ferrando A, Paddon-Jones $D$, Ronsen O, Protas E, Evans W J. Functional impact of 10 days of bed rest in healthy older adults. J Gerontol A Biol Sci Med Sci 2008;63(10):1076-1081. doi: 10.1093/ gerona/63.10.1076

20. Lourenço TM, Lernardt MH, Kletemberg DF, Seima MD, Carneiro NHK. Independência funcional em idosos longevos na admissão hospitalar. Texto Contexto - Enferm Florianópolis 2014;23(3):673-679. doi: 10.1590/010407072014001500013 